\title{
Modelo multiparche y multigrupo para la transmisión de la malaria
}

\author{
Multi-patch and multi-group model for the transmission of the malaria
}

\author{
Enith Amanda Gómez Hernández ${ }^{\text {a,* }}$ \\ Eduardo Ibargüen Mondragón ${ }^{b}$ \\ Edwin Hernando Salazar Jurado ${ }^{\text {a }}$ \\ Kernel Enrique Prieto Moreno ${ }^{c}$
}

Fecha de Recepción: 7.7.19

Fecha de Aceptación:12.12.19

DOI: https://doi.org/10.19053/01217488.v11.n1.2020.10433

\begin{abstract}
Resumen
La malaria es una enfermedad potencialmente mortal causada por parásitos transmitidos a los humanos por la picadura de mosquitos hembra infectados del género anopheles. Analizar el efecto de la movilidad y el hecho de que existen individuos que responden de manera diferente a la enfermedad puede aportar en la planeación de estrategias para evitar la propagación. Teniendo en cuenta lo anterior se propone estudiar la dinámica de la malaria considerando que los individuos pertenecen a un grupo determinado y se distribuyen en parcelas disyuntas que están conectadas de algún modo. Para esto, se plantea un modelo multiparche y multigrupo utilizando como base el modelo de Ross-Macdonald, en el cual se incorpora la movilidad desde el enfoque lagrangiano donde todos los individuos pertenecen a un grupo específico pero pasan parte de su tiempo en un número arbitrario de parches. El análisis cualitativo se realiza con base en el número reproductivo básico dando como resultado que la movilidad de las poblaciones de humanos y mosquitos divididas en grupos cambia la dinámica de la enfermedad.
\end{abstract}

Palabras clave: Análisis cualitativo, malaria, modelo multigrupo, modelo multiparche.

\begin{abstract}
Malaria is a potentially fatal disease which is caused for parasites transmitted to humans by the bite of female infected mosquitoes of the anopheles gender. Analyzing the effect of mobility and the fact that there are individuals who respond differently to the disease can contribute to the planning of strategies to prevent the spread. In view of the above, it is proposed to study the dynamics of malaria considering that individuals belong to a specific group and are distributed in disjoint plots that are connected in some way. For this, a multipatch and multigroup model is proposed using the Ross-Macdonald model as a basis, in which mobility is incorporated from the Lagrangian approach where all individuals belong to a specific group but spend part of their time in an arbitrary number of patches. The qualitative analysis is performed based on the basic reproductive number and the results showe that the mobility and dividing the population of humans and mosquitoes into groups changing the dynamics of the disease.
\end{abstract}

Key words: Qualitative analysis, malaria, multi-group model, multi-patch model.

\footnotetext{
${ }^{a}$ Est. Doctorado en Modelamiento Matemático Aplicado, Universidad Católica del Maule, Talca, Chile.

*Correo electrónico: enith.gomez.alu.ucm.cl

${ }^{b}$ Dr., Departamento de Matemáticas, Universidad de Nariño, Pasto, Colombia.

${ }^{\mathrm{c}}$ Dr., Instituto de Matemáticas, Universidad Nacional Autónoma de México, México.
} 


\section{Introducción}

La malaria es considerada una de las enfermedades más antiguas de la humanidad y en la actualidad la mitad de la población mundial está en riesgo, lo que la convierte en un problema de salud pública. En 2016 hubo 216 millones de casos distribuidos en 91 países, un aumento de aproximadamente 5 millones con respecto a 2015, que según las estimaciones costaron la vida de 445 mil personas. Entre los años 2015 y 2016 las tasas de mortalidad se mantuvieron constantes en el Sudeste Asiático, el Pacífico Occidental y África; sin embargo, aumentaron en las regiones del Mediterráneo Oriental y en América; en esta última, los países más afectados son Colombia y Brasil que reportan 36 y 37 casos de muertes, respectivamente. En este sentido la Organización Mundial de la Salud (OMS) constantemente elabora estrategias para combatirla y eliminarla, actualmente está vigente la Estrategia Técnica Mundial Contra la Malaria 2016-2030 [1, 2].

En Colombia, los departamentos más afectados por la malaria son: Chocó, Cauca, Nariño y Valle del Cauca, que registran más de diez casos por mil habitantes y agrupan el $82 \%$ de todos los notificados [3]; particularmente, Nariño aporta el $10 \%$ de los casos totales en el país y concretamente en el 2014 el departamento reportó 5175 casos de morbilidad, de los cuales el $99 \%$ corresponden a la zona del Pacífico Nariñense [4, 5].

En cuanto a la etiología de la malaria es una enfermedad causada por el parásito protozoario del género plasmodium, que se transmite al ser humano por la picadura de mosquitos hembra infectados del género anopheles [2]. Se conocen más de 175 especies de las cuales cuatro afectan al hombre: plasmodium falciparum, plasmodium vivax, plasmodium malariae y plasmodium ovale [6]. El plasmodium vivax es la causa más frecuente de la enfermedad que rara vez es mortal y el plasmodium falciparum no es común pero produce la mayoría de las muertes [7]. En Colombia, el $72 \%$ de los casos son por plasmodium vivax, el $27.1 \%$ por plasmodium falciparum y el $0.8 \%$ por malaria mixta; esta relación se invierte en los departamentos de la Costa Pacífica Colombiana, en la cual por lo menos el $80 \%$ de los casos son por plasmodium falciparum $[8,9]$.

El anopheles es un género de mosquito de la familia culicidae que habita en prácticamente todo el mundo, con especial intensidad en las zonas templadas, tropicales y subtropicales. El macho se alimenta de néctar y jugos de frutas, mientras que la hembra se alimenta principalmente de sangre. La taxonomía depende en gran medida de los orígenes geográficos, es por eso que en cada región los mosquitos tienen diferentes hábitos de alimentación, afectando de manera distinta a las personas [10].

Determinar la especie de anopheles presente en un lugar específico es esencial para estudiar las estrategias que permitan el control de la malaria, pues existen cerca de 422 especies de mosquitos, incluyendo las 70 transmisoras del agente causal de la malaria [10]. En Colombia se han encontrado alrededor de 43, de las cuales 7 son causantes de la enfermedad; los vectores primarios son: anopheles albimanus wiedemann, anopheles darlingi root y anopheles nuneztovari gabaldón; los vectores secundarios son: anopheles puntimacula dyar, anopheles pseudopunctipennis theobald, anopheles lepidotus zavortink y anopheles neivai [11].

Algunos estudios han determinado que además de los vectores primarios y secundarios reportados en [11], hay otras especies dominantes de importancia local en algunas áreas, es el caso del departamento de Nariño en el cual se distingue como vector primario al anopheles calderoni; en esta región se seleccionaron 22 localidades específicas según la incidencia histórica de la malaria, encontrando que el mosquito anopheles calderoni está en 14 localidades y se presenta en mayor abundancia en tres municipios, Boca de Prieta, Roberto Payán y Olaya Herrera. En Boca de Prieta, ejemplares fueron sometidos a ensayo inmunoenzimático (ELISA) y resultaron positivos para plasmodium falciparum $\mathrm{y}$ plasmodium vivax $[12,13]$.

La importancia de determinar que el anopheles calderoni está presente en Nariño, radica en que tiene un comportamiento diferente en relación con las otras especies encontradas en Colombia; lo más preocuparte es que la hembra se alimenta a cualquier 
hora del día, de manera que se hace evidente la necesidad de introducir herramientas adicionales dirigidas a la transmisión al aire libre para disminuir el contacto humano-vector en la Costa Pacífica Nariñense [13].

Por otra parte, la distribución espacial del mosquito anopheles en el departamento de Nariño como se muestra en la figura 1 , motiva a estudiar la malaria con subpoblaciones disyuntas de vectores y de humanos, dado que los puntos en el mapa son precisamente las regiones donde hay municipios o corregimientos como se corrobora en la figura 2, en la que además se muestra el número de casos en algunas poblaciones $[12,13]$.

Los puntos en los mapas de las figuras 1 y 2 están "conectados" dado que las personas que habitan las diferentes zonas viajan de un lugar a otro, causando que los individuos se infecten de malaria en alguno de estos sitios; también, los mosquitos pueden desplazarse de una zona a otra utilizando medios de transporte humano, lo cual podría cambiar la dinámica de la enfermedad [14].

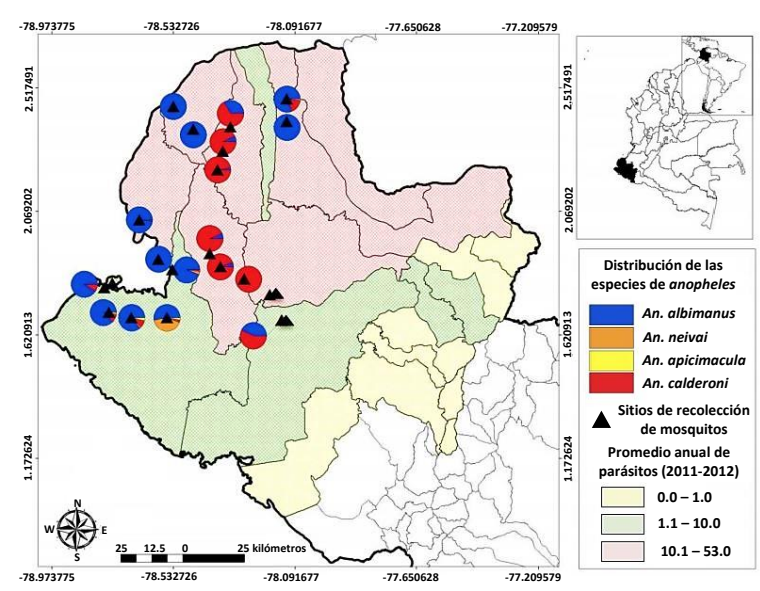

Figura 1. Distribución espacial de anopheles en el departamento de Nariño [13].

Por lo expuesto anteriormente en este artículo se propone estudiar la malaria con un modelo en parches dada la distribución mostrada en las figuras 1 y 2. Además, se consideran diferentes grupos con la intención de dividir las poblaciones dependiendo del impacto de la enfermedad, por ejemplo, diferenciar los humanos según la edad o por estratos socioeconómicos y los vectores entre mosquitos resistentes o

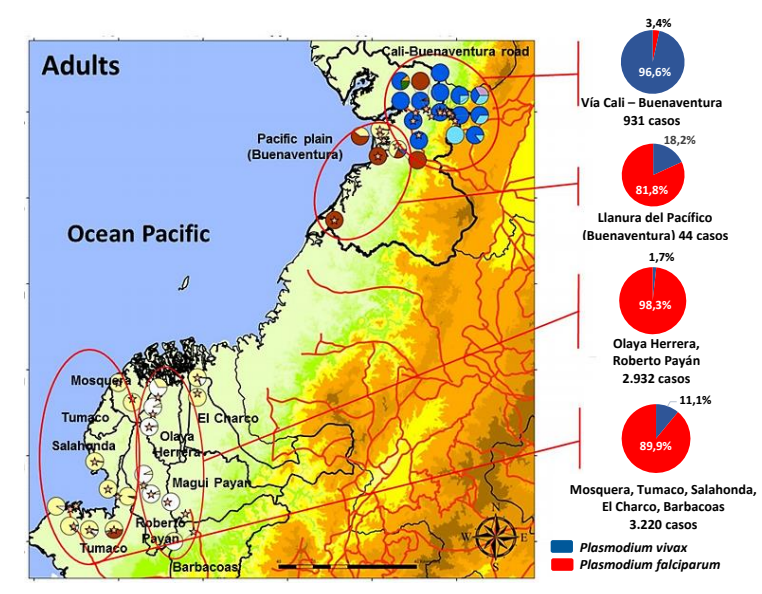

Figura 2. Distribución de especies de anopheles en el sur de la Costa Pacífica Colombiana y casos reportados de malaria [12].

no resistentes a los insecticidas. Así, en este artículo, se estudia la dinámica de la malaria considerando que los individuos pertenecen a un grupo determinado y se distribuyen en parcelas disyuntas que están conectadas de algún modo, para lo cual se plantea un modelo multiparche y multigrupo utilizando el modelo de Ross-Macdonald para la transmisión de la malaria, en el cual se incorpora la movilidad desde un enfoque lagrangiano, donde todos los individuos pertenecen a un grupo específico, pero pasan parte de su tiempo en un número arbitrario de parches.

\section{Metodología}

Para el desarrollo del presente escrito se tiene en cuenta que hay una región en Colombia, la Costa Pacífica Nariñense que aporta cifras significativas de la enfermedad en el país; es una zona donde no sólo el vector primario tiene un comportamiento diferente que afecta las medidas que sugieren las entidades de salud para disminuir el contacto con el mosquito, sino también, la mayoría de los casos de malaria son por plasmodium falciparum, patógeno que causa más muertes.

Además, la distribución espacial que tiene la población y el hecho de que en la literatura se puede encontrar diversos modelos en ecuaciones diferenciales ordinarias para la dinámica de la malaria, donde se considera que la población esta distribuida homogéneamente sin considerar la movilidad ni los grupos [15, 16, 17, 18, 19, 20, 21], propicia la idea de estudiar el efecto de estos aspectos en 
un modelo clásico como el de Ross-Macdonald. Una base fundamental para formular el modelo se encuentra desarrollada en el artículo [22], en el cual proponen un modelo multiparche y multigrupo para la dinámica SEIRS.

El análisis del modelo se desarrolla de acuerdo a la teoría de los sistemas dinámicos desarrollada en [23], en la cual se establece la condición para que un sistema de ecuaciones diferenciales ordinarias genere un flujo fuertemente monótono, principalmente consiste en que la matriz jacobiana del campo vectorial debe ser irreducible en cada punto, resultado que se puede aplicar para mostrar que la órbita genérica de un sistema irreducible converge al equilibrio. La hipótesis de la irreducibilidad también permite establecer que la estabilidad de un equilibrio está determinada por el valor propio dominante de la matriz jacobiana, cuando ésta es cuasi-positiva.

\section{Resultados y discusión}

\subsection{Modelo de Ross-Macdonald}

Entre los modelos matemáticos existentes para la malaria, el modelo de Ross y Macdonald ha sentado las bases de la mayoría de los modelos epidemiológicos actuales, dado que estudia la dinámica de manera "sencilla" y se ajusta al fenómeno biológico [24]. El modelo describe los cambios en los humanos infectados y los mosquitos portadores, la población susceptible se modela implícitamente ya que el tamaño de las poblaciones se mantiene constante.

Los supuestos para el modelo son: $N$ el tamaño de la población de humanos, $\bar{x}$ la proporción de humanos infectados, $M$ el tamaño de la población de mosquitos, $\bar{y}$ la proporción de mosquitos infectados, $\ell=M / N$ el número de mosquitos por persona, $a$ el número de picaduras de mosquitos por humano por unidad de tiempo, $b$ la proporción de picaduras necesarias para que un mosquito transmita la infección, $c$ la probabilidad de infección de mosquitos, $\gamma$ la tasa de recuperación por persona infecciosa y $\mu$ la tasa de mortalidad de los mosquitos. Con lo anterior se obtiene el siguiente sistema de ecuaciones diferenciales

$$
\begin{aligned}
& \frac{d \bar{x}}{d t}=a b \ell \bar{y}(1-\bar{x})-\gamma \bar{x} \\
& \frac{d \bar{y}}{d t}=a c \bar{x}(1-\bar{y})-\mu \bar{y}
\end{aligned}
$$

En este modelo se supone que la tasa de mortalidad de los seres humanos es insignificante cuando se compara con su tasa de recuperación, y la tasa de recuperación de los mosquitos es despreciable cuando se compara con su tasa de mortalidad. El equilibrio libre de la enfermedad $E_{0}=(0,0)$ es estable o inestable dependiendo del número reproductivo básico $\mathscr{R}_{0}$, el cual se calcula con el operador de la siguiente generación y está dado por

$$
\mathscr{R}_{0}=\sqrt{\frac{a^{2} b c \ell}{\mu \gamma}}
$$

Luego si $\mathscr{R}_{0}<1$, la enfermedad desaparece y $E_{0}$ es asintóticamente estable. $\mathrm{Si} \mathscr{R}_{0}>1$, el equilibrio $E_{0}$ es inestable y hay un punto de equilibrio endémico con coordenadas

$$
\bar{x}=\frac{a^{2} c b \ell-\gamma \mu}{a c(r+a b \ell)}, \quad \bar{y}=\frac{a^{2} c b \ell-\gamma \mu}{a b m(a c+\mu)}
$$

que es asintóticamente estable [24].

\subsection{Modelo de Ross-Macdonald en grupos y en parches}

Para formular el modelo en parches y en grupos, es necesario precisar que el número de individuos no necesariamente es el mismo en cada grupo, por lo que se define el modelo de Ross-Macdonald con números absolutos de humanos y mosquitos, para lo cual se hace el cambio de variable $x=N \bar{x}$ e $y=M \bar{y}$, obteniéndose

$$
\begin{aligned}
& \frac{d x}{d t}=\left(\frac{a}{N} b\right) y(N-x)-\gamma x \\
& \frac{d y}{d t}=\left(\frac{a}{N} c\right) x(M-y)-\mu y
\end{aligned}
$$

Los mosquitos incrementan el número de picaduras por humano por unidad de tiempo en forma lineal cuando la población de humanos es pequeña; sin embargo, a medida que crece la población de personas la tasa de picaduras decrece, por lo tanto se puede relacionar el número de picaduras de mosquitos $a$ con el tamaño de la población de humanos $N$. En el 


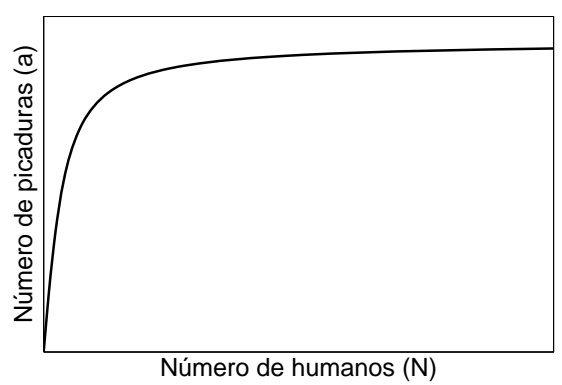

Figura 3. Forma hipotética del número de picaduras en función del número de humanos [25].

artículo [25] proponen una función $a=g(N)$, donde $g(N)$ puede ajustarse a la curva de la figura 3 .

Por el comportamiento de $g(N)$, en el artículo [25] proponen que el número de picaduras por humano por unidad de tiempo $a$ es proporcional al tamaño de la población $N$ para bajas densidades de personas, es decir $a=\kappa N$ donde $\kappa$ es la constante de proporcionalidad; si se supone además que la probabilidad $b$ de que un humano se infecte es igual a la probabilidad $c$ de que un mosquito pase a ser portador, como lo sugieren en el artículo [26], se obtiene $\kappa b=\kappa c=\alpha$, por lo tanto el modelo (4) puede reescribirse como

$$
\begin{aligned}
& \frac{d x}{d t}=\alpha y(N-x)-\gamma x \\
& \frac{d y}{d t}=\alpha x(M-y)-\mu y
\end{aligned}
$$

Ahora, se considera que la población está estructurada de forma arbitraria en $u$ grupos que interactúan en $v$ parches, cada grupo tiene la dinámica del modelo (5), $x_{i}$ e $y_{i}$ representan la población de humanos y mosquitos infectados del grupo $i$ con $i=1, \ldots, u$; los humanos susceptibles e infectados del grupo $i$ permanecen una proporción de tiempo $p_{i j}$ y $s_{i j}$, respectivamente en el parche $j$ con $j=1, \ldots, v$; los mosquitos no portadores y portadores del grupo $i$ permanecen una proporción de tiempo $r_{i j}$ y $q_{i j}$, respectivamente en el parche $j$. La población de humanos en el parche $j$ en un tiempo $t$ está dada por

$$
\widehat{N}_{j}=\sum_{k=1}^{u}\left(p_{k j}\left(N_{k}-x_{k}\right)+s_{k j} x_{k}\right)
$$

y la población de mosquitos en el parche $j$ en un tiempo $t$ está dada por

$$
\widehat{M}_{j}=\sum_{k=1}^{u}\left(r_{k j}\left(M_{k}-y_{k}\right)+q_{k j} y\right)
$$

Las poblaciones descritas en (6) y (7) describen la dinámica temporal de cada una de las poblaciones en el parche $j$, ponderada por los patrones de movilidad de cada grupo y cada estado epidemiológico. La población de humanos infectados y de mosquitos portadores es $\sum_{k=1}^{u} s_{k j} x_{k}$ y $\sum_{k=1}^{u} q_{k j} y_{k}$, respectivamente; por lo tanto la proporción de infectados en el parche $j$ está dada por

$$
\begin{aligned}
N_{j}^{*} & =\frac{\sum_{k=1}^{u} s_{k j} x_{k}}{\sum_{k=1}^{u}\left(p_{k j}\left(N_{k}-x_{k}\right)+s_{k j} x_{k}\right)} \\
M_{j}^{*} & =\frac{\sum_{k=1}^{u} q_{k j} y_{k}}{\sum_{k=1}^{u}\left(r_{k j}\left(M_{k}-y_{k}\right)+q_{k j} y_{k}\right)}
\end{aligned}
$$

Los individuos susceptibles del grupo $i$ pueden infectarse en cualquier parche $j$, durante la permanencia en ese lugar, por lo tanto la dinámica en $u$ grupos y $v$ parches es

$$
\begin{aligned}
\frac{d x_{i}}{d t} & =\sum_{j=1}^{v} \alpha_{j} p_{i j}\left(N_{i}-x_{i}\right) M_{j}^{*}-\gamma_{i} x_{i} \\
\frac{d y_{i}}{d t} & =\sum_{j=1}^{v} \alpha_{j} r_{i j}\left(M_{i}-y_{i}\right) N_{j}^{*}-\mu_{i} y_{i}
\end{aligned}
$$

En el modelo (8) se estructura las poblaciones en un número arbitrario de grupos; estas poblaciones, con diferentes estados epidemiológicos, pasan cierto tiempo en algún parche, donde pueden infectarse o infectar a otros. En cada ubicación se tiene un riesgo particular de infección vinculado a sus condiciones ambientales.

En particular los humanos susceptibles del grupo $i, N_{i}-x_{i}$ se infectan cuando están en contacto con la proporción de mosquitos portadores del parche $j$, a una tasa $\alpha_{j}$ asociada al riesgo de contraer la enfermedad en ese parche; la tasa de recuperación de los humanos infectados en el grupo $i$ es $\gamma_{i}$, independientemente del lugar. Una explicación similar se puede dar para la infección de mosquitos susceptibles del grupo $i$. El esquema de la dinámica se muestra en la figura 4.

Con el propósito de analizar la dinámica del modelo multiparche y multigrupo, se reescribe (8) de la forma

$$
\begin{aligned}
\dot{\boldsymbol{x}}= & \operatorname{diag}(\boldsymbol{N}-\boldsymbol{x}) \mathbb{P} \operatorname{diag}(\boldsymbol{\alpha}) \operatorname{diag}\left(\boldsymbol{v}_{\mathbf{1}}\right)^{-1} \mathbb{Q}^{T} \boldsymbol{y} \\
& -\operatorname{diag}(\boldsymbol{\gamma}) \boldsymbol{x} \\
\dot{\boldsymbol{y}}= & \operatorname{diag}(\boldsymbol{M}-\boldsymbol{y}) \mathbb{R} \operatorname{diag}(\boldsymbol{\alpha}) \operatorname{diag}\left(\boldsymbol{v}_{\mathbf{2}}\right)^{-1} \mathbb{S}^{T} \boldsymbol{x} \\
& -\operatorname{diag}(\boldsymbol{\mu}) \boldsymbol{y}
\end{aligned}
$$




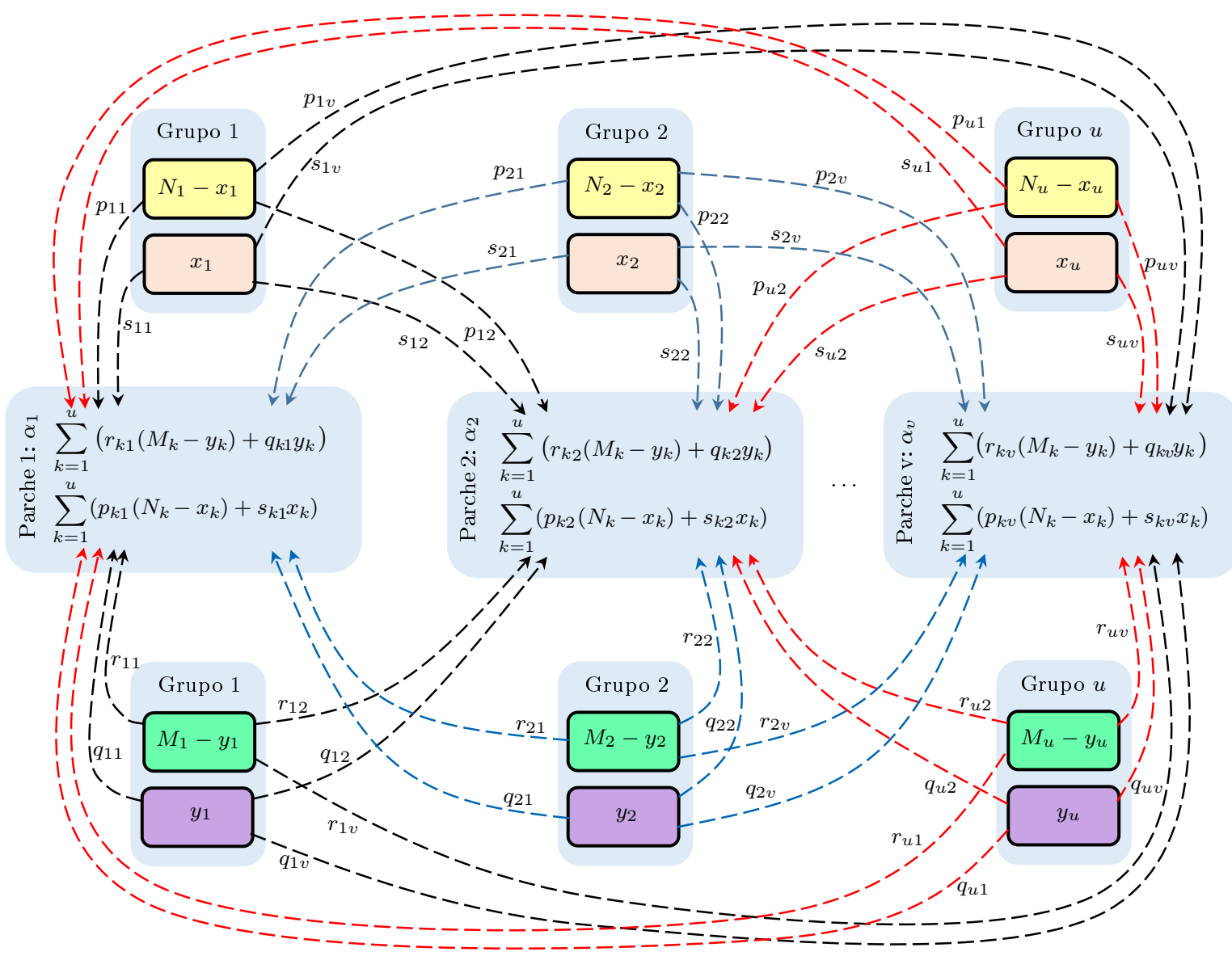

Figura 4. Esquema de la dinámica del modelo (8).

donde, $\quad \boldsymbol{x}=\left[x_{1}, \ldots, x_{u}\right]^{T}, \quad \boldsymbol{y}=\left[y_{1}, \ldots, y_{u}\right]^{T}$, $\boldsymbol{N}=\left[N_{1}, \ldots, N_{u}\right]^{T}, \quad \boldsymbol{M}=\left[M_{1}, \ldots, M_{u}\right]^{T}, \quad \boldsymbol{\gamma}=$ $\left[\gamma_{1}, \ldots, \gamma_{u}\right]^{T}, \boldsymbol{\mu}=\left[\mu_{1}, \ldots, \mu_{u}\right]^{T}, \boldsymbol{\alpha}=\left[\alpha_{1}, \ldots, \alpha_{v}\right]^{T}$ $, \mathbb{P}=\left(p_{i j}\right), \mathbb{Q}=\left(q_{i j}\right), \mathbb{R}=\left(r_{i j}\right)$ y $\mathbb{S}=\left(s_{i j}\right)$, con $1 \leq i \leq u$ y $1 \leq j \leq v, \boldsymbol{v}_{\mathbf{1}}=\mathbb{R}^{T}(\boldsymbol{M}-\boldsymbol{y})+\mathbb{Q}^{T} \boldsymbol{y}$, $\boldsymbol{v}_{\mathbf{2}}=\mathbb{P}^{T}(\boldsymbol{N}-\boldsymbol{x})+\mathbb{S}^{T} \boldsymbol{x}$.

Para el análisis del modelo (9) es necesario que la matriz $\mathbb{P} \mathbb{Q}^{T} \mathbb{R} \mathbb{S}^{T}$ sea irreducible, esto significa que con una condición inicial de un mosquito portador o de un humano infectado en cualquier sitio, la enfermedad es capaz de extenderse por todos los grupos y los parches, dado que el grafo asociado a la matriz es fuertemente conectado y ningún lugar queda excepto del contacto humano-vector.

\subsection{Número reproductivo básico}

Para calcular el número de reproductivo básico $\mathscr{R}_{0}$, es necesario descomponer los compartimentos infectados de (9) como una suma de los términos de infección $\mathscr{F}$ y los términos de transición $\mathscr{V}$ como se describe en [24], es decir

$$
\begin{aligned}
{\left[\begin{array}{c}
\dot{\boldsymbol{x}} \\
\dot{\boldsymbol{y}}
\end{array}\right] } & =\mathscr{F}(\boldsymbol{x}, \boldsymbol{y})-\mathscr{V}(\boldsymbol{x}, \boldsymbol{y}) \\
& =\left[\begin{array}{l}
\operatorname{diag}(\boldsymbol{N}-\boldsymbol{x}) \mathbb{P} \operatorname{diag}(\boldsymbol{\alpha}) \operatorname{diag}\left(\boldsymbol{v}_{\mathbf{1}}\right)^{-1} \mathbb{Q}^{T} \boldsymbol{y} \\
\operatorname{diag}(\boldsymbol{M}-\boldsymbol{y}) \mathbb{R} \operatorname{diag}(\boldsymbol{\alpha}) \operatorname{diag}\left(\boldsymbol{v}_{\mathbf{2}}\right)^{-1} \mathbb{S}^{T} \boldsymbol{x}
\end{array}\right] \\
& -\left[\begin{array}{l}
\operatorname{diag}(\boldsymbol{\gamma}) \boldsymbol{x} \\
\operatorname{diag}(\boldsymbol{\mu}) \boldsymbol{y}
\end{array}\right]
\end{aligned}
$$

La matriz jacobiana de $\mathscr{F}(\boldsymbol{x}, \boldsymbol{y})$ es

$$
F=D \mathscr{F}(\boldsymbol{x}, \boldsymbol{y})=\left[\begin{array}{ll}
a_{11} & a_{12} \\
a_{21} & a_{22}
\end{array}\right]
$$

donde,

$$
\begin{aligned}
a_{11}= & -\operatorname{diag}(\mathbf{1}) \mathbb{P} \operatorname{diag}(\boldsymbol{\alpha})\left(\operatorname{diag}\left(\boldsymbol{v}_{\mathbf{1}}\right)\right)^{-1} \mathbb{Q}^{T} \boldsymbol{y} \\
a_{22}= & -\operatorname{diag}(\mathbf{1}) \mathbb{R} \operatorname{diag}(\boldsymbol{\alpha})\left(\operatorname{diag}\left(\boldsymbol{v}_{\mathbf{2}}\right)\right)^{-1} \mathbb{S}^{T} \boldsymbol{x} \\
a_{12}= & \operatorname{diag}(\boldsymbol{N}-\boldsymbol{x}) \mathbb{P} \operatorname{diag}(\boldsymbol{\alpha})\left(\operatorname{diag}\left(\boldsymbol{v}_{\mathbf{1}}\right)\right)^{-1} \mathbb{Q}^{T} \\
- & \left(\operatorname{diag}(\boldsymbol{N}-\boldsymbol{x}) \mathbb{P} \operatorname{diag}(\boldsymbol{\alpha})\left(\left(\operatorname{diag}\left(\boldsymbol{v}_{\mathbf{1}}\right)\right)^{2}\right)^{-1}\right) \\
& \left(\operatorname{diag}\left(-\mathbb{R}^{T}(\mathbf{1})+\mathbb{Q}^{T}(\mathbf{1})\right) \mathbb{Q}^{T} \boldsymbol{y}\right)
\end{aligned}
$$




$$
\begin{aligned}
a_{21}= & \operatorname{diag}(\boldsymbol{M}-\boldsymbol{y}) \mathbb{R} \operatorname{diag}(\boldsymbol{\alpha})\left(\operatorname{diag}\left(\boldsymbol{v}_{\mathbf{2}}\right)\right)^{-1} \mathbb{S}^{T} \\
- & \left(\operatorname{diag}(\boldsymbol{M}-\boldsymbol{y}) \mathbb{R} \operatorname{diag}(\boldsymbol{\alpha})\left(\left(\operatorname{diag}\left(\boldsymbol{v}_{\mathbf{2}}\right)\right)^{2}\right)^{-1}\right) \\
& \left(\operatorname{diag}\left(-\mathbb{P}^{T}(\mathbf{1})+\mathbb{S}^{T}(\mathbf{1})\right) \mathbb{S}^{T} \boldsymbol{y}\right)
\end{aligned}
$$

Evaluando la matriz (10) en el punto de equilibrio $E_{0}=(\mathbf{0}, \mathbf{0})$ se tiene

$$
F(\mathbf{0}, \mathbf{0})=\left[\begin{array}{cc}
\mathbf{0}_{u \times u} & \bar{a}_{12} \\
\bar{a}_{21} & \mathbf{0}_{u \times u}
\end{array}\right]
$$

donde,

$$
\begin{aligned}
& \bar{a}_{12}=\operatorname{diag}(\boldsymbol{N}) \mathbb{P} \operatorname{diag}(\boldsymbol{\alpha}) \operatorname{diag}\left(\mathbb{R}^{T} \boldsymbol{M}\right)^{-1} \mathbb{Q}^{T} \\
& \bar{a}_{21}=\operatorname{diag}(\boldsymbol{M}) \mathbb{R} \operatorname{diag}(\boldsymbol{\alpha}) \operatorname{diag}\left(\mathbb{P}^{T} \boldsymbol{N}\right)^{-1} \mathbb{S}^{T}
\end{aligned}
$$

La matriz jacobiana de $\mathscr{V}$ está dada por

$$
V=D \mathscr{V}(\boldsymbol{x}, \boldsymbol{y})=\left[\begin{array}{cc}
\operatorname{diag}(\boldsymbol{\gamma}) & \mathbf{0}_{u \times u} \\
\mathbf{0}_{u \times u} & \operatorname{diag}(\boldsymbol{\mu})
\end{array}\right]=D \mathscr{V}(\mathbf{0}, \mathbf{0})
$$

Ahora, como $V$ es invertible se puede calcular $F V^{-1}$

$$
F V^{-1}=\left[\begin{array}{cc}
\mathbf{0}_{u \times u} & \bar{a}_{12} \operatorname{diag}(\mathbf{1} / \boldsymbol{\mu}) \\
\bar{a}_{21} \operatorname{diag}(\mathbf{1} / \boldsymbol{\gamma}) & \mathbf{0}_{u \times u}
\end{array}\right]
$$

El polinomio característico de $F V^{-1}$ está dado por

$$
\operatorname{det}\left(\lambda^{2} I-\bar{a}_{12} \operatorname{diag}(\mathbf{1} / \boldsymbol{\mu}) \bar{a}_{21} \operatorname{diag}(\mathbf{1} / \boldsymbol{\gamma})\right)=0
$$

Por lo tanto, el número reproductivo básico $\mathscr{R}_{0}$ es

$$
\mathscr{R}_{0}(u, v)=\left[\rho\left(\bar{a}_{12} \operatorname{diag}(\mathbf{1} / \boldsymbol{\mu}) \bar{a}_{21} \operatorname{diag}(\mathbf{1} / \boldsymbol{\gamma})\right)\right]^{\frac{1}{2}}
$$

La ecuación (11) representa el número de infecciones secundarias ocasionadas por la llegada de una persona infectada a una población de susceptibles.

\subsection{Efectos de la heterogeneidad en el número reproductivo básico}

En este apartado se estudia los efectos que tiene la proporción de tiempo que pasan los individuos de un grupo $i$ en algún parche $j$, además la influencia del número de grupos y de parches en el número reproductivo básico (11).

Particularmente, el tiempo de permanencia de los individuos en algún parche influye en el brote epidémico o en la extinción de la enfermedad, para mostrar que esto puede suceder se supone el modelo
(8) en un grupo y dos parches, con su respectivo número reproductivo básico $\mathscr{R}_{0}(1,2)$ expresado como sigue

$$
\begin{aligned}
& {\left[\frac{\alpha^{2}}{\mu \gamma}\left[p_{11} \quad p_{12}\right]\left[\begin{array}{cc}
1 / r_{11} & 0 \\
0 & 1 / r_{12}
\end{array}\right]\left[\begin{array}{l}
q_{11} \\
q_{12}
\end{array}\right]\right.} \\
& \left.\left[r_{11} r_{12}\right]\left[\begin{array}{cc}
1 / p_{11} & 0 \\
0 & 1 / p_{12}
\end{array}\right]\left[\begin{array}{l}
s_{11} \\
s_{12}
\end{array}\right]\right]^{\frac{1}{2}}
\end{aligned}
$$

Además, si se supone que el número reproductivo básico en un parche y un grupo es menor que uno, se puede observar el efecto de las matrices $\mathbb{P}, \mathbb{Q}, \mathbb{R}$ y $\mathbb{S}$ con el siguiente ejemplo.

Sea $\mathscr{R}_{0}(1,1)=0,55$, entonces $\frac{\alpha^{2}}{\mu \gamma}=0,3$ y reemplazando este valor en el número reproductivo básico del modelo (8) en un grupo y dos parches con la siguiente opción para las matrices de tiempo de residencia $\mathbb{P}_{1}=(1 / 32 / 3)$, $\mathbb{Q}_{1}=(1 / 43 / 4), \mathbb{R}_{1}=(1 / 21 / 2)$ y $\mathbb{S}_{1}=(1 / 54 / 5)$ se tiene $\mathscr{R}_{0}(1,2)=0,59$. Sin embargo, escogiendo $\mathbb{P}_{2}=\left(\begin{array}{lll}3 / 4 & 1 / 4\end{array}\right), \mathbb{Q}_{2}=\left(\begin{array}{lll}7 / 8 & 1 / 8\end{array}\right)$, $\mathbb{R}_{2}=(4 / 1612 / 16)$ y $\mathbb{S}_{2}=(3 / 74 / 7)$, se obtiene $\mathscr{R}_{0}(1,2)=1,35$.

Lo anterior muestra que si la dinámica tiene varios parches, el tiempo de permanencia de los individuos puede repercutir en un brote epidémico en el modelo (8), es decir $\mathscr{R}_{0}(1,2)>1$; aunque el número reproductivo básico $\mathscr{R}_{0}(1,1)$ sea menor que uno.

A continuación se presenta un resultado formal de como $\mathscr{R}_{0}(u, v)$ cambia dependiendo del número de parches y de grupos, para lo cual se define el número reproductivo básico de un grupo específico $i$ como

$$
\mathscr{R}_{0}^{i}=\left(\frac{1}{\gamma_{i} \mu_{i}} \sum_{k=1}^{v} \alpha_{k}^{2} q_{i k} s_{i k}\right)^{1 / 2}
$$

Teorema 3.1. Se tienen las siguientes desigualdades

1. $\operatorname{mín}_{1, \ldots, u} \mathscr{R}_{0}^{i} \leq \mathscr{R}_{0}(u, v) \leq \operatorname{máx}_{1, \ldots, u} \mathscr{R}_{0}^{i}$.

2. $\mathscr{R}_{0}(u, v) \geq \mathscr{R}_{0}(1, v) \geq \mathscr{R}_{0}(1,1)$.

3. Para un número fijo de grupos $u, \mathscr{R}_{0}(u, v) \geq$ $\mathscr{R}_{0}\left(u, v^{\prime}\right)$ donde $v$ y $v^{\prime}$ son enteros tal que $v \geq$ $v^{\prime}$. 
Demostración.

1. El número reproductivo básico del modelo (8) está dado por

$$
\begin{aligned}
\mathscr{R}_{0}^{2}(u, v)=\rho & \left(\operatorname{diag}(\boldsymbol{N}) \mathbb{P} \operatorname{diag}(\boldsymbol{\alpha}) \operatorname{diag}\left(\mathbb{R}^{T} \boldsymbol{M}\right)^{-1} \mathbb{Q}^{T}\right. \\
& \operatorname{diag}(\mathbf{1} / \boldsymbol{\mu}) \operatorname{diag}(\boldsymbol{M}) \mathbb{R} \operatorname{diag}(\boldsymbol{\alpha}) \\
& \left.\operatorname{diag}\left(\mathbb{P}^{T} \boldsymbol{N}\right)^{-1} \mathbb{S}^{T} \operatorname{diag}(\mathbf{1} / \boldsymbol{\gamma})\right)
\end{aligned}
$$

un elemento de la matriz es

$$
h_{i j}=\frac{1}{\gamma_{j} \mu_{j}} \sum_{k=1}^{v} \alpha_{k}^{2} p_{i k} q_{j k} r_{i k} s_{j k} N_{i} M_{i} \frac{1}{\sum_{l=1}^{u} p_{l k} r_{l k} M_{l} N_{l}}
$$

Si el producto de las matrices $\mathbb{P} \mathbb{Q}^{T} \mathbb{R S}^{T}$ es irreducible, la matriz

$$
\begin{array}{r}
\operatorname{diag}(\boldsymbol{N}) \mathbb{P} \operatorname{diag}(\boldsymbol{\alpha}) \operatorname{diag}\left(\mathbb{R}^{T} \boldsymbol{M}\right)^{-1} \mathbb{Q}^{T} \operatorname{diag}(\mathbf{1} / \boldsymbol{\mu}) \\
\operatorname{diag}(\boldsymbol{M}) \mathbb{R} \operatorname{diag}(\boldsymbol{\alpha}) \operatorname{diag}\left(\mathbb{P}^{T} \boldsymbol{N}\right)^{-1} \mathbb{S}^{T} \operatorname{diag}(\mathbf{1} / \boldsymbol{\gamma})
\end{array}
$$

es irreducible, entonces el radio espectral satisface la inecuación de Frobenius de acuerdo a [22]

$$
\operatorname{mín}_{j} h_{j} \leq \mathscr{R}_{0}^{2} \leq \operatorname{máx}_{j} h_{j}
$$

donde $h_{j}=\sum_{i=1}^{u} h_{i j}$, luego

$$
\begin{aligned}
h_{j} & =\sum_{i=1}^{u} h_{i j} \\
& =\sum_{i=1}^{u} \frac{1}{\gamma_{j} \mu_{j}} \sum_{k=1}^{v} \alpha_{k}^{2} p_{i k} q_{j k} r_{i k} s_{j k} N_{i} M_{i} \frac{1}{\sum_{l=1}^{u} p_{l k} r_{l k} M_{l} N_{l}} \\
& =\frac{1}{\gamma_{j} \mu_{j}} \sum_{k=1}^{v} \alpha_{k}^{2} q_{j k} s_{j k} \frac{\sum_{i=1}^{u} p_{i k} r_{i k} N_{i} M_{i}}{\sum_{l=1}^{u} p_{l k} r_{l k} M_{l} N_{l}} \\
& =\frac{1}{\gamma_{j} \mu_{j}} \sum_{k=1}^{v} \alpha_{k}^{2} q_{j k} s_{j k} \\
& =\left(\mathscr{R}_{0}^{j}\right)^{2}
\end{aligned}
$$

de (13) se obtiene

$$
\operatorname{mín}_{i}\left(\mathscr{R}_{0}^{i}\right)^{2} \leq \mathscr{R}_{0}^{2}(u, v) \leq \operatorname{máx}_{i}\left(\mathscr{R}_{0}^{i}\right)^{2}
$$

por lo tanto

$$
\operatorname{mín}_{i} \mathscr{R}_{0}^{i} \leq \mathscr{R}_{0}(u, v) \leq \operatorname{máx}_{i} \mathscr{R}_{0}^{i}
$$

2. Usando la inecuación del item 1 se tiene

$$
\begin{aligned}
\mathscr{R}_{0}(u, v) & \geq \operatorname{mín}_{i=1, \ldots, u} \mathscr{R}_{0}^{i} \\
& :=\mathscr{R}_{0}(1, v)
\end{aligned}
$$

Además,

$$
\begin{aligned}
\mathscr{R}_{0}(1, v) & =\mathscr{R}_{0}^{1} \\
& =\left(\frac{1}{\gamma_{1} \mu_{1}} \sum_{k=1}^{v} \alpha_{k}^{2} q_{1 k} s_{1 k}\right)^{1 / 2} \\
& \geq\left(\frac{1}{\gamma_{1} \mu_{1}} \alpha_{1}^{2} q_{11} s_{11}\right)^{1 / 2} \\
& :=\mathscr{R}_{0}(1,1)
\end{aligned}
$$

3. Si el número de grupos es fijo en el número reproductivo básico de la ecuación (11), los parámetros son los mismos para cualquier cantidad de parches, así la demostración se reduce a comparar $Z_{v}$ y $Z_{v^{\prime}}$ definidas a continuación. Para $v$ parches se tiene

$$
Z_{v}^{i j}=\sum_{k=1}^{v} \alpha_{k}^{2} p_{i k} q_{j k} r_{i k} s_{j k} N_{i} M_{i} \frac{1}{\sum_{l=1}^{u} p_{l k} r_{l k} M_{l} N_{l}}
$$

Para $v^{\prime}$ parches se tiene

$$
Z_{v^{\prime}}^{i j}=\sum_{k=1}^{v^{\prime}} \alpha_{k}^{2} p_{i k} q_{j k} r_{i k} s_{j k} N_{i} M_{i} \frac{1}{\sum_{l=1}^{u} p_{l k} r_{l k} M_{l} N_{l}}
$$

Así, para $v \geq v^{\prime}$, se tiene que $Z_{v}^{i j} \geq Z_{v^{\prime}}^{i j}$. Por lo tanto $\mathscr{R}_{0}(u, v) \geq \mathscr{R}_{0}\left(u, v^{\prime}\right)$ por el teorema de PerronFrobenius (ver teorema 3.1 en [23], p. 60).

\subsection{Estabilidad global del punto de equilibrio libre de infección}

A continuación, usando el Principio de Invarianza de LaSalle (ver corolario 3.1 en [27], p. 116) y el teorema de Lyapunov (ver teorema 3 en [28], p. 131) se prueba la estabilidad asintótica global de la solución de equilibrio libre de infección $E_{0}$.

Teorema 3.2. Si $\mathscr{R}_{0}(u, v) \leq 1$ entonces $E_{0}$ es global y asintóticamente estable, siempre que el producto de las matrices $\mathbb{P} \mathbb{Q}^{T} \mathbb{R} \mathbb{S}^{T}$ sea irreducible.

Demostración. Sea $\left(\omega_{x}, \omega_{y}\right)$ un valor propio izquierdo asociado a la matriz

$$
\begin{aligned}
& \operatorname{diag}(\boldsymbol{N}) \mathbb{P} \operatorname{diag}(\boldsymbol{\alpha}) \operatorname{diag}\left(\mathbb{R}^{T} \boldsymbol{M}\right)^{-1} \mathbb{Q}^{T} \operatorname{diag}(\mathbf{1} / \boldsymbol{\mu}) \\
& \operatorname{diag}(\boldsymbol{M}) \mathbb{R} \operatorname{diag}(\boldsymbol{\alpha}) \operatorname{diag}\left(\mathbb{P}^{T} \boldsymbol{N}\right)^{-1} \mathbb{S}^{T} \operatorname{diag}(\mathbf{1} / \boldsymbol{\gamma})=\mathbb{A}
\end{aligned}
$$

luego,

$$
\begin{aligned}
\left(\omega_{x}, \omega_{y}\right) \mathbb{A} & =\left(\omega_{x}, \omega_{y}\right)[\rho(A)]^{1 / 2} \\
& =\left(\omega_{x}, \omega_{y}\right) \rho\left(-F V^{-1}\right)
\end{aligned}
$$


como el producto de las matrices $\mathbb{P} \mathbb{Q}^{T} \mathbb{R} \mathbb{S}^{T}$ es irreducible, entonces la matriz $\mathbb{A}$ es irreducible, lo cual implica que $\left(\omega_{x}, \omega_{y}\right) \gg 0($ ver teorema 3.1 en [23], p. 60). Entonces

$$
V(\boldsymbol{x}, \boldsymbol{y})=\left(\omega_{\boldsymbol{x}}, \omega_{\boldsymbol{y}}\right)\left[\begin{array}{cc}
\operatorname{diag}(\mathbf{1} / \boldsymbol{\gamma}) & \mathbf{0}_{u \times u} \\
\mathbf{0}_{u \times u} & \operatorname{diag}(\mathbf{1} / \boldsymbol{\mu})
\end{array}\right]\left[\begin{array}{l}
\boldsymbol{x} \\
\boldsymbol{y}
\end{array}\right]
$$

es una función de Lyapunov que tiene como derivada orbital a

$$
\dot{V}=\left(\omega_{\boldsymbol{x}}, \omega_{\boldsymbol{y}}\right)\left[\begin{array}{cc}
\operatorname{diag}(\mathbf{1} / \boldsymbol{\gamma}) & \mathbf{0}_{u \times u} \\
\mathbf{0}_{u \times u} & \operatorname{diag}(\mathbf{1} / \boldsymbol{\mu})
\end{array}\right]\left[\begin{array}{c}
\dot{\boldsymbol{x}} \\
\dot{\boldsymbol{y}}
\end{array}\right]
$$

donde,

$$
\begin{aligned}
\dot{\boldsymbol{x}}= & \operatorname{diag}(\boldsymbol{N}-\boldsymbol{x}) \mathbb{P} \operatorname{diag}(\boldsymbol{\alpha}) \operatorname{diag}\left(\mathbb{R}^{T}(\boldsymbol{M}-\boldsymbol{y})+\mathbb{Q}^{T} \boldsymbol{y}\right)^{-1} \mathbb{Q}^{T} \boldsymbol{y} \\
& -\operatorname{diag}(\boldsymbol{\gamma}) \boldsymbol{x} \\
\dot{\boldsymbol{y}}= & \operatorname{diag}(\boldsymbol{M}-\boldsymbol{y}) \mathbb{R} \operatorname{diag}(\boldsymbol{\alpha}) \operatorname{diag}\left(\mathbb{P}^{T}(\boldsymbol{N}-\boldsymbol{x})+\mathbb{S}^{T} \boldsymbol{x}\right)^{-1} \mathbb{S}^{T} \boldsymbol{x} \\
& -\operatorname{diag}(\boldsymbol{\mu}) \boldsymbol{y}
\end{aligned}
$$

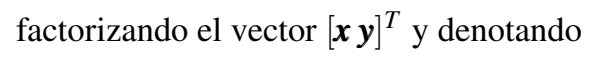

$$
\begin{aligned}
& \operatorname{diag}(\boldsymbol{N}) \mathbb{P} \operatorname{diag}(\boldsymbol{\alpha}) \operatorname{diag}\left(\mathbb{R}^{T} \boldsymbol{M}\right)^{-1} \mathbb{Q}^{T}= \bar{a}_{12} \\
& \operatorname{diag}(\boldsymbol{M}) \mathbb{R} \operatorname{diag}(\boldsymbol{\alpha}) \operatorname{diag}\left(\mathbb{P}^{T} \boldsymbol{N}\right)^{-1} \mathbb{S}^{T}=\bar{a}_{21}
\end{aligned}
$$

se tiene que la derivada orbital $\dot{V}$ puede ser expresada como

$$
\begin{aligned}
& \dot{V}=\left(\omega_{\boldsymbol{x}}, \omega_{\boldsymbol{y}}\right)\left[\begin{array}{cc}
-I_{u} & \bar{a}_{12} \operatorname{diag}(\mathbf{1} / \boldsymbol{\gamma}) \\
\bar{a}_{21} \operatorname{diag}(\mathbf{1} / \boldsymbol{\mu}) & -I_{u}
\end{array}\right]\left[\begin{array}{l}
\boldsymbol{x} \\
\boldsymbol{y}
\end{array}\right] \\
&=\left(\omega_{\boldsymbol{x}}, \omega_{\boldsymbol{y}}\right)\left(V^{-1} F-I\right)\left[\begin{array}{l}
\boldsymbol{x} \\
\boldsymbol{y}
\end{array}\right] \\
&=\left(\mathscr{R}_{0}-1\right)\left(\omega_{\boldsymbol{x}}, \omega_{\boldsymbol{y}}\right)\left[\begin{array}{l}
\boldsymbol{x} \\
\boldsymbol{y}
\end{array}\right] \\
& \leq 0
\end{aligned}
$$

Ahora para probar que es asintóticamente estable, se debe demostrar que ninguna solución de (8) que entra a la región $\mathscr{S}=\left\{(\boldsymbol{x}, \boldsymbol{y}) \in \mathbb{R}^{2 u}: \dot{V}(\boldsymbol{x}, \boldsymbol{y})=0\right\}$ permanece allí indefinidamente salvo la solución trivial, según el Principio de LaSalle, la única posibilidad es que $\mathscr{S}=$ $\left\{(\boldsymbol{x}, \boldsymbol{y}) \in R^{2 u}: \boldsymbol{x}=\mathbf{0} \wedge \boldsymbol{y}=\mathbf{0}\right\}$, es decir, $E_{0}$ es el único punto de $\mathscr{S}$, entonces $E_{0}$ es global y asintóticamente estable.

\subsection{Estabilidad del equilibrio endémico}

Determinar la existencia del equilibrio endémico puede ser laborioso, de hecho en el artículo [22] muestran que las no linealidades intrincadas del modelo (8) pueden conllevar a multiples equilibrios endémicos, por tal razón se estudia la dinámica en un caso particular, en el cual el riesgo de contraer la enfermedad depende de los tamaños de cada una de las poblaciones, más específicamente se supone que el riesgo de infección en cada parche es proporcional al tamaño de la población del mismo, esto es

$$
\begin{aligned}
\alpha_{j} \widehat{N}_{j} & =\alpha_{j} \sum_{k=1}^{u}\left(p_{k j}\left(N_{k}-x_{k}\right)+s_{k j} x_{k}\right) \\
\alpha_{j} \widehat{M}_{j} & =\alpha_{j} \sum_{k=1}^{u}\left(r_{k j}\left(M_{k}-y_{k}\right)+q_{k j} y\right)
\end{aligned}
$$

Así, la tasas con que los individuos susceptibles humanos y mosquitos pasan a ser infectados en el parche $j$ son

$$
\begin{gathered}
\alpha_{j} \widehat{N}_{j} \frac{\sum_{k=1}^{u} s_{k j} x_{k}}{\sum_{k=1}^{u}\left(p_{k j}\left(N_{k}-x_{k}\right)+s_{k j} x_{k}\right)}:=\alpha_{j} \sum_{k=1}^{u} s_{k j} x_{k} \\
\alpha_{j} \widehat{M}_{j} \frac{\sum_{k=1}^{u} q_{k j} y_{k}}{\sum_{k=1}^{u}\left(r_{k j}\left(M_{k}-y_{k}\right)+q_{k j} y_{k}\right)}:=\alpha_{j} \sum_{k=1}^{u} q_{k j} y_{k}
\end{gathered}
$$

Por lo tanto, el modelo (8) queda expresado como

$$
\begin{aligned}
\frac{d x_{i}}{d t} & =\sum_{j=1}^{v} \alpha_{j} p_{i j}\left(N_{i}-x_{i}\right) \sum_{k=1}^{u} q_{k j} y_{k}-\gamma_{i} x_{i} \\
\frac{d y_{i}}{d t} & =\sum_{j=1}^{v} \alpha_{j} r_{i j}\left(M_{i}-y_{i}\right) \sum_{k=1}^{u} s_{k j} x_{k}-\mu_{i} y_{i}
\end{aligned}
$$

Reescribiendo el sistema dinámico (14) en forma matricial se tiene

$$
\begin{aligned}
& \dot{\boldsymbol{x}}=\operatorname{diag}(\boldsymbol{N}-\boldsymbol{x}) \mathbb{P} \operatorname{diag}(\boldsymbol{\alpha}) \mathbb{Q}^{T} \boldsymbol{y}-\operatorname{diag}(\boldsymbol{\gamma}) \boldsymbol{x} \\
& \dot{\boldsymbol{y}}=\operatorname{diag}(\boldsymbol{M}-\boldsymbol{y}) \mathbb{R} \operatorname{diag}(\boldsymbol{\alpha}) \mathbb{S}^{T} \boldsymbol{x}-\operatorname{diag}(\boldsymbol{\mu}) \boldsymbol{y}
\end{aligned}
$$

El número reproductivo básico del modelo (15) es

$$
\begin{aligned}
\mathscr{R}_{0}^{D D}= & {\left[\boldsymbol { \rho } \left(\operatorname{diag}(\boldsymbol{N}) \mathbb{P} \operatorname{diag}(\boldsymbol{\alpha}) \mathbb{Q}^{T} \operatorname{diag}(\mathbf{1} / \boldsymbol{\mu})\right.\right.} \\
& \left.\left.\operatorname{diag}(\boldsymbol{M}) \mathbb{R} \operatorname{diag}(\boldsymbol{\alpha}) \mathbb{S}^{T} \operatorname{diag}(\mathbf{1} / \boldsymbol{\gamma})\right)\right]^{1 / 2}
\end{aligned}
$$

que se obtiene de (11).

El siguiente teorema muestra la existencia y la estabilidad del equilibrio endémico del modelo (14), para obtener el resultado se utiliza el módulo de estabilidad $s(J)$ definido por

$$
s(J)=\operatorname{máx}\{\operatorname{Re}(\lambda): \lambda \in \sigma(J)\}
$$

donde $\operatorname{Re}(\lambda)$ es la parte real del valor propio $\lambda \mathrm{y}$ $\sigma(J)$ es el conjunto de los valores propios de la matriz jacobiana $J, s(J)$ determina las propiedades de estabilidad de los equilibrios, puesto que si el máximo de los valores propios es negativo los otros también lo son. Además se puede relacionar con el número reproductivo básico $\mathscr{R}_{0}^{D D}$ de acuerdo al artículo [29], en el cual mencionan y demuestran los siguientes items 

i. $\mathscr{R}_{0}^{D D}<1$ si y sólo si $s(J)<0$.
ii. $\mathscr{R}_{0}^{D D}=1$ si y sólo si $s(J)=0$.
iii. $\mathscr{R}_{0}^{D D}>1$ si y sólo si $s(J)>0$.

Teorema 3.3. Existe un único equilibrio endémico que es local y asintóticamente estable cuando $\mathscr{R}_{0}^{D D}>1$.

Demostración. La demostración se realiza con la teoría de los sistemas irreducibles encontrada en el capítulo 4 del libro [23], para lo cual se necesita que el sistema (15) sea estrictamente monótono; es decir, que la matriz jacobiana (16) sea irreducible (ver teorema 1.1 en [23], p.56).

$$
J=\left[\begin{array}{ll}
A_{1} & A_{2} \\
A_{3} & A_{4}
\end{array}\right]
$$

donde

$$
\begin{aligned}
& A_{1}=-\mathbb{P} \operatorname{diag}(\boldsymbol{\alpha}) \mathbb{Q}^{T} \boldsymbol{y}-\operatorname{diag}(\boldsymbol{\gamma}) \\
& A_{2}=\operatorname{diag}(\boldsymbol{N}-\boldsymbol{x}) \mathbb{P} \operatorname{diag}(\boldsymbol{\alpha}) \mathbb{Q}^{T} \\
& A_{3}=\operatorname{diag}(\boldsymbol{M}-\boldsymbol{y}) \mathbb{R} \operatorname{diag}(\boldsymbol{\alpha}) \mathbb{S}^{T} \\
& A_{4}=-\mathbb{R} \operatorname{diag}(\boldsymbol{\alpha}) \mathbb{S}^{T} \boldsymbol{x}-\operatorname{diag}(\boldsymbol{\mu})
\end{aligned}
$$

La matriz (16) es irreducible si el grafo asociado es fuertemente conectado (ver definición 2.6 en [30], p. 30), lo cual es cierto si se supone que $(\boldsymbol{x}, \boldsymbol{y}) \neq(\boldsymbol{N}, \boldsymbol{M})$ y que un solo individuo infectado puede causar la infección en todos los parches.

Por otro lado, sea $f(\boldsymbol{x}, \boldsymbol{y})$ el campo vectorial descrito por (15), entonces

$$
f(\boldsymbol{N}, \boldsymbol{M})=\left(\begin{array}{c}
-\operatorname{diag}(\boldsymbol{\gamma}) \boldsymbol{N} \\
-\operatorname{diag}(\boldsymbol{\mu}) \boldsymbol{M}
\end{array}\right) \ll \mathbf{0}
$$

luego, el flujo $\phi_{t}(\boldsymbol{N}, \boldsymbol{M})$ converge monótonamente a un equilibrio para cualquier $(\boldsymbol{N}, \boldsymbol{M})$ (ver proposición 2.1 en [23], p. 34). Ahora se prueba la existencia de un equilibrio endémico utilizando la solución trivial $(\mathbf{0 , 0})$ y la matriz jacobiana evaluada en este punto

$$
\left[\begin{array}{cc}
-\operatorname{diag}(\boldsymbol{\gamma}) & \operatorname{diag}(\boldsymbol{N}) \mathbb{P} \operatorname{diag}(\boldsymbol{\alpha}) \mathbb{Q}^{T} \\
\operatorname{diag}(\boldsymbol{M}) \mathbb{R} \operatorname{diag}(\boldsymbol{\alpha}) \mathbb{S}^{T} & -\operatorname{diag}(\boldsymbol{\mu})
\end{array}\right]
$$

Como el sistema es estrictamente monótono no tiene órbitas periódicas, luego para que exista un punto endémico basta con que el número reproductivo básico $\mathscr{R}_{0}^{D D}$ sea mayor que uno, lo que implica que el módulo de estabilidad $s(J)>0$, garantizando al menos un valor propio positivo, así $E_{0}$ es inestable y por lo tanto existe al menos un equilibrio positivo $E_{1}$ de el sistema (14) (ver corolario 3.2 en [23], p. 60)

Ahora, para probar que el sistema (14) tiene un único equilibrio, se supone que existe otro, $E_{2}$. Se debe demostrar que $E_{1}=E_{2}$, se argumenta por contradicción. Sea $E_{1} \neq E_{2}$, sin pérdida de generalidad

$$
E_{1}<E_{2}
$$

Dado que $E_{1}$ y $E_{2}$ son puntos de equilibrio satisfacen el sistema

$$
\left[\begin{array}{cc}
-\operatorname{diag}(\boldsymbol{\gamma}) & A_{2} \\
A_{3} & -\operatorname{diag}(\boldsymbol{\mu})
\end{array}\right]\left[\begin{array}{l}
\boldsymbol{x} \\
\boldsymbol{y}
\end{array}\right]=\left[\begin{array}{l}
\mathbf{0} \\
\mathbf{0}
\end{array}\right]
$$

Se observa que el sistema anterior es de la forma $A \mathbf{x}=\mathbf{0}$, luego $s(A)$ es cero, por lo tanto $A\left(E_{1}\right)$ y $A\left(E_{2}\right)$ tienen el mismo módulo de estabilidad. Sin embargo, como $A$ es cuasi-positiva, es decir las componentes son no negativas excepto las de la diagonal principal, entonces $s\left(A\left(E_{2}\right)\right)<s\left(A\left(E_{1}\right)\right)$ (ver corolario 3.2 en [23], p. 60), lo cual es una contradicción; en consecuencia únicamente existe un equilibrio distinto de $E_{0}$.

Finalmente se demuestra que $E_{1}=(\overline{\boldsymbol{x}}, \overline{\boldsymbol{y}})$ es local $\mathrm{y}$ asintóticamente estable, para lo cual se denota

$$
\begin{aligned}
& B_{1}=-\mathbb{P} \operatorname{diag}(\boldsymbol{\alpha}) \mathbb{Q}^{T} \overline{\boldsymbol{y}}-\operatorname{diag}(\boldsymbol{\gamma}) \\
& B_{2}=\operatorname{diag}(\boldsymbol{N}-\overline{\boldsymbol{x}}) \mathbb{P} \operatorname{diag}(\boldsymbol{\alpha}) \mathbb{Q}^{T} \\
& B_{3}=\operatorname{diag}(\boldsymbol{M}-\overline{\boldsymbol{y}}) \mathbb{R} \operatorname{diag}(\boldsymbol{\alpha}) \mathbb{S}^{T} \\
& B_{4}=-\mathbb{R} \operatorname{diag}(\boldsymbol{\alpha}) \mathbb{S}^{T} \overline{\boldsymbol{x}}-\operatorname{diag}(\boldsymbol{\mu})
\end{aligned}
$$

y se verifica la siguiente desigualdad

$$
\begin{aligned}
J\left(E_{1}\right) & =\left[\begin{array}{ll}
B 1 & B_{2} \\
B 3 & B 4
\end{array}\right] \\
& <\left[\begin{array}{cc}
-\operatorname{diag}(\boldsymbol{\gamma}) & A_{2} \\
A_{3} & -\operatorname{diag}(\boldsymbol{\mu})
\end{array}\right] \\
& =A
\end{aligned}
$$

es decir, $s(J)<s(A)$. Como $A E_{1}=\mathbf{0}$, dado que $E_{1}$ es un punto de equilibrio entonces $s(A)=0$, lo cual implica que el módulo de estabilidad de la matriz jacobiana es menor que cero, garantizando que todos los valores propios de la matriz de la linealización son negativos; por lo tanto, $E_{1}$ es local y asintóticamente estable. 


\section{Simulación numérica}

Las simulaciones numéricas permiten visualizar el comportamiento de las soluciones haciendo uso de los métodos numéricos, con lo cual se obtiene soluciones aproximadas. En la figura 5 se muestra la solución numérica para un caso particular del modelo (8), en un tiempo de 500 días con condición inicial (20,0); el valor de la tasa de recuperación de los humanos es $\gamma_{i}=0,0029$ y la tasa de mortalidad de los mosquitos es $\mu_{i}=0,0039$, los cuales son tomados de [31]; el riesgo de infección es $\alpha_{j}=0,03$ y las matrices de permanencia para dos parches son $\mathbb{P}=\mathbb{Q}=\mathbb{R}=\mathbb{S}=(1 / 10$ 9/10). En la simulación con un grupo y dos parches (figura de la derecha) se observa que la población de infectados crece en menos tiempo en relación con el crecimiento en un parche y un grupo (figura de la izquierda), lo cual indica que $\mathscr{R}_{0}(1,2)$ es mayor que $\mathscr{R}_{0}(1,1)$ que está en concordancia con el teorema 3.1, donde se ve el efecto del número de parches y grupos en el $\mathscr{R}_{0}(u, v)$.
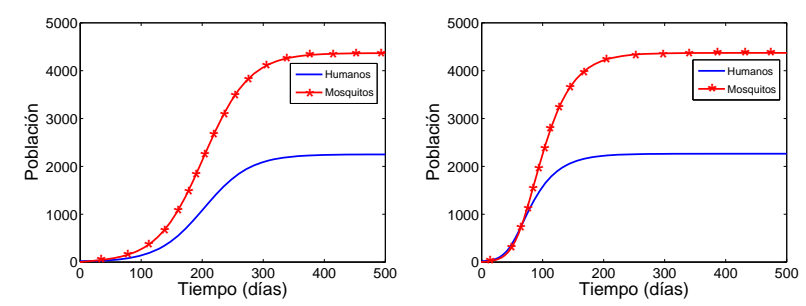

Figura 5. Solución numérica del modelo (8): imagen izquierda, un grupo y un parche; imagen derecha, un grupo y dos parches.

En la figura 6 se muestra la solución numérica en dos parches y en dos grupos con los mismos parámetros de 5 y con las siguientes matrices de permanencia

$$
\begin{aligned}
& \mathbb{P}=\mathbb{Q}=\mathbb{S}=\left[\begin{array}{ll}
1 / 10 & 9 / 10 \\
1 / 10 & 9 / 10
\end{array}\right] \\
& \mathbb{R}=\left[\begin{array}{ll}
1 / 10 & 9 / 10 \\
9 / 10 & 1 / 10
\end{array}\right]
\end{aligned}
$$

Se supuso la matriz de permanencia $\mathbb{R}$ distinta a las demás para obtener dos grupos con dinámicas diferentes, en los cuales se observa que la población de cada grupo crece en menor tiempo con respecto a la simulación 5 , es decir, $\mathscr{R}_{0}(2,2)$ es mayor que $\mathscr{R}_{0}(1,2)$ que está de acuerdo a lo demostrado en el teorema 3.1.
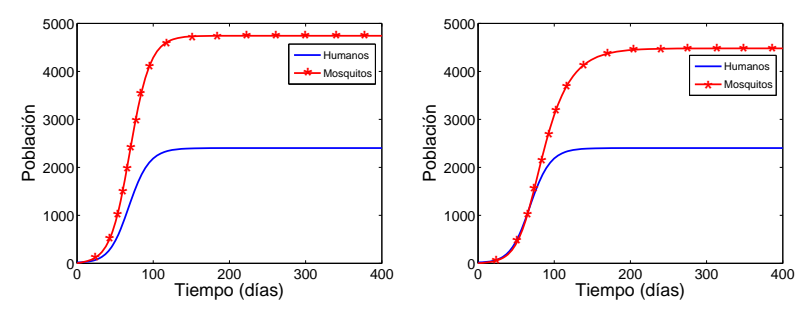

Figura 6. Solución numérica del modelo (8) en dos parches: imagen izquierda, grupo 1; imagen derecha, grupo 2.

\section{Conclusiones}

El modelo matemático de Ross-Macdonald extendido a parches y a grupos permite inferir los siguientes resultados.

- Si el producto de las matrices de permanencia no son irreducibles el sistema dinámico se desacopla y el problema se reduce a estudiar el comportamiento de cada parche.

- El número reproductivo básico $\mathscr{R}_{0}(u, v)$ depende de la permanencia de los individuos en las diferentes zonas.

- Si el producto de las matrices de permanencia es irreducible, entonces el número reproductivo básico en $u$ grupos y en $v$ parches está acotado por el mínimo y el máximo $\mathscr{R}_{0}^{i}$ de los grupos.

- Si se fija la cantidad de grupos, el número reproductivo básico $\mathscr{R}_{0}(u, v)$ incrementa a medida que aumenta el número de parches.

- Si el número reproductivo básico $\mathscr{R}_{0}(u, v) \leq 1$ el equilibrio libre de infección $E_{0}$ es global y asintóticamente estable, es decir la enfermedad se extingue.

- En el caso de que la tasa de infección asociada al parche dependa del tamaño de la población, existe un equilibrio endémico $E_{1}$ que es local y asintóticamente estable cuando $\mathscr{R}_{0}^{D D}(u, v)>1$, lo cual indica que la enfermedad persiste.

El análisis del modelo propuesto evidencia que la movilidad y los grupos influyen en la dinámica de transmisión de la malaria y aunque el resultado es teórico muestra características de la problemática; por lo cual, se puede sugerir que se empleen estrategias que tengan en cuenta la migración de la población. Un procedimiento que se puede seguir para disminuir el número de casos es identificar un 
foco de infección y aislarlo hasta que pueda ser controlado.

\section{Referencias}

[1] Organización Mundial de la Salud, Estrategia técnica mundial contra la malaria 2016-2030 [online], Organización Mundial de la Salud, 2017 Disponible en: http://apps.who.int/iris/bitstream/10665/ 186671/1/9789243564999_spa.pdf

[2] World Health Organization, World malaria report 2016 [online], World Health Organization, 2017 Disponible en: http://apps.who.int/iris/bitstream/10665/ 259492/1/9789241565523-eng.pdf

[3] J. Rodríguez, "Dinámica probabilista temporal de la epidemia de malaria en Colombia", Revista Med, vol. 17, no. 2, pp. 214-221, 2009.

[4] Instituto Departamental de Salud de Nariño, El IDSN conmemora desde Tumaco el día mundial de lucha contra el paludismo o malaria: invertir en el futuro, derrotar el paludismo [online], 2014 Disponible en: http://www.idsn.gov.co/index.php/noticias/ 952-dia-mundial-del-paludismo-25-de-abril-de-20॥13]

[5] Instituto Departamental de Salud de Nariño. Boletín epidemiológico: Situación de las enfermedades transmitidas por vectores ETV en el departamento de nariño, año 2014, 2015 Disponible en: http://www.idsn.gov.co/ index.php/subdireccion-de-salud-publica/ epidemiologia/boletin-epidemiologico/ 1846-boletin-etv-ano-2014

[6] M. Carballo, "Diseño, síntesis y evaluación biológica de nuevos derivados de 1, 4-di-n-Óxido de quinoxalina como agentes antimaláricos y antileishmaniásicos", Tesis de Maestría, Facultad de Farmacia, Universidad de Navarra, Pamplona, 2010.

[7] C. García, "Características demográficas de la población en función de su exposición de la malaria en la amazonía ecuatoriana", Tesis B.S, Facultad de Medicina, Universidad San Francisco de Quito, Quito, 2010.

[8] L. Molineros, O. Calvache, H. Bolaños, C. Castillo y C. Torres, "Aplicaciones de un modelo integral para el estudio de la malaria urbana en San Andrés de Tumaco, Colombia”, Revista Cubana de Medicina Tropical, vol. 66, no. 1, pp. 3-19, 2014.

[9] Instituto Nacional de Salud-Vigilancía y Control en Salud Pública, Protocolo de vigilancia y control de la malaria [online], 2010, Disponible en: https: //www.minsalud.gov.co/comunicadosPrensa/ Documents/MALARIA.pdf

[10] M. Wahlgren y P. Perlmann, Malaria: molecular and clinical aspects, CRC Press, 1 edition, 2003.

[11] Organización Panamerica de la Salud-Instituto Nacional de Salud. Gestión para la vigilancia entomológica y control de la transmisión de malaria [online], 2016, Disponible en: http:// www.paho.org/hq/

[12] M. Ahumada, L. Orjuela, P. Pareja, M. Conde, D. Cabarcas y E. Cubillos, "Spatial distributions of anopheles species in relation to malaria incidence at 70 localities in the highly endemic northwest and south pacific coast regions of colombia", Malaria journal, vol. 15, no. 407, pp. 1-16, 2016.

[13] L. Orjuela, M. Ahumada, I. Avila, S. Herrera, J. Beier y M. Quiñones, "Human biting activity, spatial-temporal distribution and malaria vector role of anopheles calderoni in the southwest of colombia”, Malaria journal, vol. 14, no. 256, pp. 1-9, 2015.

[14] C. Blanco, "Factores socio ambientales y demográficos asociados a la transmisión de la malaria en los barrios de Jericó y Cristales en la ciudad Trujillo Colon", Tesis de Maestría, Facultad de Ciencias Médicas, Universidad Nacional Autónoma de Honduras, Honduras, 2006.

[15] J. P. Romero-Leiton, J. M. Aguilar y E. Ibargüen, "An optimal control problem applied to malaria disease in Colombia". Applied Mathematical Sciences, vol. 12, no. 6, pp. 279-292, 2018

[16] D. L. Smith y F. E. McKenzie, "Statics and dynamics of malaria infection", Anopheles mosquitoes. Malaria journal, vol. 3, no. 1, pp. 13, 2004.

[17] J. P. Leiton, J. M. Montoya, M. Villaroel y E. Ibargüen, "Influencia de la fuerza de infección 
y la transmisión vertical en la malaria: Modelado Matemático", Revista Facultad de Ciencias Básicas, vol. 13, no. 1, pp. 4-18, 2017.

[18] J. J. Tewa, R. Fokouop, B. Mewoli y S. Bowong, "Mathematical analysis of a general class of ordinary differential equations coming from within-hosts models of malaria with immune effectors", Applied Mathematics and Computation, vol. 218, no. 14, pp. 7347-7361, 2012.

[19] J. P. Romero-Leiton, J. E. Castellanos y E. Ibargüen-Mondragón, "An optimal control problem and cost-effectiveness analysis of malaria disease with vertical transmission applied to San Andrés de Tumaco (Colombia)", Сomputational and Applied Mathematics, vol. 38, no. 133, 2019.

[20] J. P. Romero-Leiton y E. Ibargüen-Mondragón, "Stability analysis and optimal control intervention strategies of a malaria mathematical model",Applied Sciences, vol. 21, pp. 184-218, 2019.

[21] E. A. Gómez-Hernández y E. IbargüenMondragón, "A two patch model for the population dynamics of mosquito-borne diseases", Journal of Physics: Conference Series, vol. 1408, 2019.

[22] D. Bichara, y A. Iggidr, "Multi-patch and multigroup epidemic models: a new framework", Journal of Mathematical Biology, vol. 77, no. 1, pp. 107-134, 2017.

[23] H. Smith, Monotone Dynamical Systems, American Mathematical Society, vol. 41, 1995
[24] F. Brauer, D. Chowell, C. Castillo, B. Espinoza y E. De la Pava, Modelos de la propagación de enfermedades infecciosas, Universidad Auntónoma de Occidente, vol. 1, 2015.

[25] M. Basáñez y D. Rodríguez, "Dinámica de transmisión y modelos matemáticos en enfermedades transmitidas por vectores". Entomotropica, vol. 19, no. 3, pp. 1-22, 2004.

[26] S. Ruan, D. Xiao y J. Beier, "On the delayed ross-macdonald model for malaria transmission”, Bull Math Biol, vol. 70, no. 4, pp. 10981114, 2008.

[27] H. Khali, Nonlinear Systems. Prentice-Hall, segunda edición, 1996.

[28] L. Perko, Differential equations and dynamical systems, Springer Science \& Business Media, vol. 7, 3 edition, 2013.

[29] P. Driessche y J. Watmough, "Reproduction numbers and sub-threshold endemic equilibria for compartmental models of disease transmission”, Mathematical Biosciences, vol. 180, no. 1-2, pp. 29-48, 2002.

[30] A. Berman y R. J. Plemmons, Nonnegative matrices in the mathematical sciences, Siam, vol. 9, 1994.

[31] J. Romero, J. Montoya, M. Villaroel y E. Ibargüen, "Influencia de la fuerza de infección y la transmisión vertical en la malaria: modelado matemático", Revista Facultad de Ciencias Básicas, vol. 13, no. 1, pp. 4-18, 2017. 